\title{
Vitamin D nutritional status and the risk for cardiovascular disease (Review)
}

\author{
MIN LIU ${ }^{1}$, XIANCHI LI ${ }^{2}$, RONGRONG SUN ${ }^{3}$, YI ZENG $^{3}$, SHUANG CHEN $^{2}$ and PEIYING ZHANG ${ }^{2}$ \\ ${ }^{1}$ Department of Cardiology, Xuzhou Clinical School of Xuzhou Medical College; ${ }^{2}$ Department of Cardiology, \\ Xuzhou Central Hospital, The Affiliated Xuzhou Hospital of Medical College of Southeast University; \\ ${ }^{3}$ Xuzhou Clinical Medical College of Nanjing University of Chinese Medicine, Xuzhou, Jiangsu 221009, P.R. China
}

Received November 9, 2015; Accepted February 5, 2016

DOI: $10.3892 /$ etm.2016.3047

\begin{abstract}
Cardiovascular disease (CVD) is the leading cause of morbidity and mortality. CVD has a significant impact on health care systems worldwide and over 23 million individuals are expected to succumb to the disease by 2030 . Early onset of atherosclerosis in childhood along with other risk factors of CVD, including elevated circulating lipids, have been shown to persist in adulthood and lead to CVD. Vitamin D deficiency is considered a risk factor for the pathogenesis of CVD, with childhood nutritional status of vitamin $\mathrm{D}$ being an important determinant of the development of CVD. Low levels of 25-hydroxyvitamin D can arise due to reduced intake as well as geographical location, and other diseases/conditions such as chronic kidney disease and obesity. Childhood vitamin D deficiency can progress and lead to atherosclerosis and other CVDs in adulthood. Early intervention with vitamin D supplementation is an ideal approach towards preventive therapy. However, there is no clear consensus regarding the role of vitamin D in childhood CVD. In the present study, we reviewed the available evidence in favor of and against such a role for this vitamin.
\end{abstract}

\section{Contents}

1. Introduction

2. Vitamin D deficiency and disturbed hormonal balance

3. The association of vitamin D and cardiovascular disease

4. Inflammation and vitamin D status

5. Vitamin D and metabolic disease

6. Vitamin deficiency in chronic kidney disease

Correspondence to: Dr Peiying Zhang, Department of Cardiology, Xuzhou Central Hospital, The Affiliated Xuzhou Hospital of Medical College of Southeast University, 199 South Jiefang Road, Xuzhou, Jiangsu 221009, P.R. China

E-mail: zpying58@126.com

Key words: vitamin D, cardiovascular disease, atherosclerosis

\section{Introduction}

Cardiovascular disease (CVD) is the major cause of mortality with the exception of cancer and has a significant impact on health care systems and the economy of several countries worldwide, with $>23$ million individuals expected to succumb to CVD annually by 2030 (1). CVD is also the leading cause of morbidity and mortality among patients with chronic kidney disease (CKD). These patients exhibit cardiovascular complications such as abnormal arterial compliance, elevated left ventricular mass, and diastolic dysfunction (2). Early onset of atherosclerosis in childhood (3) along with other risk factors of CVD, such as elevated circulating lipids have been shown to persist in adulthood and lead to disease (4). Several studies (5-8) have suggested the possible role of vitamin D deficiency as a risk factor for the pathogenesis of CVD and considered the childhood nutritional status of vitamin D as an important determinant of the development of CVD. However, there is no clear consensus regarding the role of vitamin D in childhood CVD. Thus, we reviewed the available evidence in favor of and against such a role for this vitamin.

Vitamin D is a fat-soluble vitamin, derived primarily from the conversion of sterols in our skin, a reaction promoted by sunlight radiation. The most well-known action of vitamin D is on the calcium homeostasis and bone metabolism, and disturbances in this process are associated with rickets in children and osteomalacia in adults (5). Vitamin D inhibits the release of calcitonin and parathyroid hormone $(\mathrm{PTH})$ from the thyroid and parathyroid glands. The concerted action of these two hormones and vitamin $\mathrm{D}$ regulates the intestinal absorption of calcium and renal calcium and phosphate metabolism and the overall bone metabolism.

Several indirect associations have been identified between vitamin D status and different forms of CVD (Fig. 1). Thus, a higher mortality rate due to ischemic heart disease was previously observed during winter months (6). Similarly, deaths due to ischemic heart disease were elevated at higher latitudes, whereas this mortality was decreased in areas closer to the equator (7). The association between vitamin D and CVDs indicated that in adults, lower circulating 25-hydroxyvitamin D levels are associated with metabolic syndrome, obesity, hypertension, diabetes, myocardial infarction, stroke and overall cardiovascular death (8). A stronger association has 


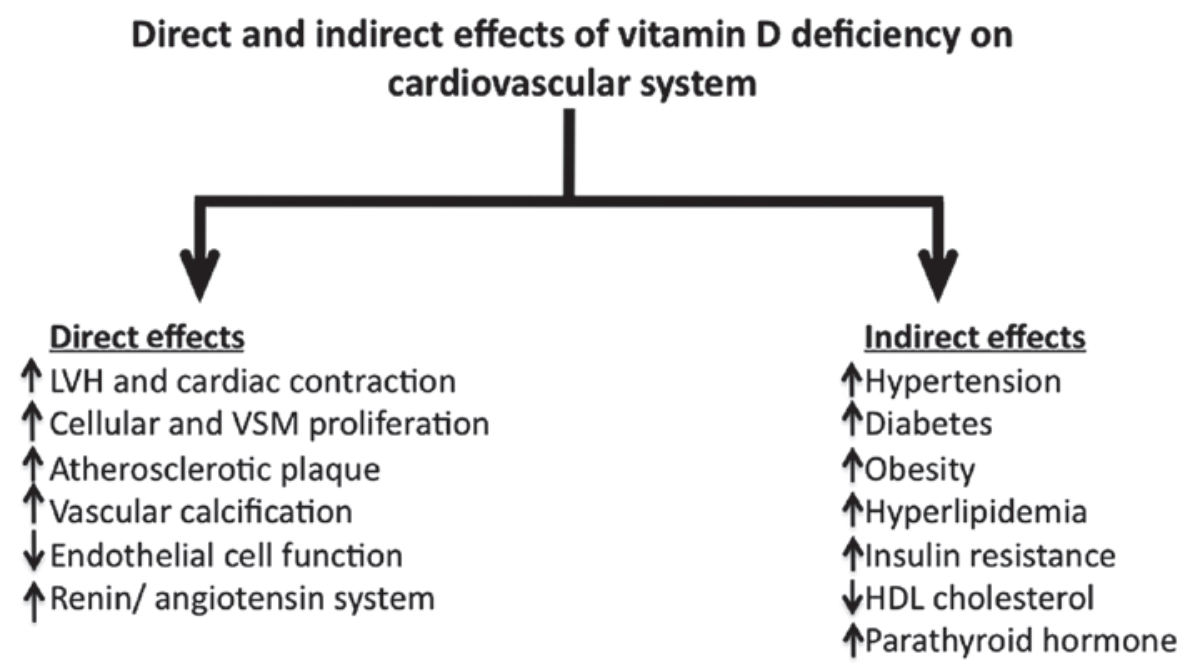

Figure 1. Direct and indirect effects of vitamin D deficiency on cardiovascular system. Vitamin D deficiency has several direct and indirect effects on cardiovascular system that have wide-ranging effects on heart function and these effects are associated with several other metabolic disturbances such as diabetes, obesity and hypertension. LVH, left ventricular hypertrophy; VSM, vascular smooth muscle; HDL, high-density lipoprotein.

been identified between lower 25-hydroxyvitamin D levels and CVD risk factors such as obesity, elevated systolic blood pressure, decreased high-density lipoprotein (HDL) cholesterol, and insulin resistance in older children and adolescents (9).

\section{Vitamin D deficiency and disturbed hormonal balance}

Many hormone systems and associated pathways are impacted by vitamin $D$ and these in turn influence cardiovascular function through a number of mechanisms. The downregulation of PTH is an important factor by which vitamin $\mathrm{D}$ can have a positive effect on heart function, as chronic vitamin D deficiency leads to the over production of $\mathrm{PTH}$, which causes left ventricular hypertrophy (LVH), valvular calcification, myocardial calcification, cardiac arrhythmia and arterial hypertension (10). Deficiency of vitamin D also leads to increased inflammation, endothelial dysfunction and upregulation of the renin-angiotensin system (RAS), which is known to play an important role in controlling blood pressure, intravascular volume and electrolyte balance. Vitamin D receptor knockout mice were found to have hyperactive RAS together with high blood pressure and cardiac hypertrophy (11). There was also an inverse correlation between 1,25-dihydroxyvitamin D3 levels in blood and RAS function and blood pressure in normal and hypertensive subjects (12). Besides affecting heart function through hormonal systems, vitamin D has been shown to influence the cardiomyocyte cell cycle (13) through its receptor, and to suppress cardiac cell proliferation and to protect the structure and function of cardiomyocytes (14).

\section{The association of vitamin D and CVD}

In as much as elevated circulating levels of childhood non-HDL cholesterol (or total cholesterol minus HDL cholesterol) have been found to be associated with an increased risk of atherosclerosis-related mortality in adults (15), childhood non-HDL cholesterol is considered an important marker for CVD risk by the National Heart Lung and Blood Institute (Bethesda, Maryland, USA). Recently, it was shown that higher 25-hydroxyvitamin D levels are associated with lower non-HDL cholesterol in children and it has been suggested that early intervention in children with vitamin D supplementation can help reduce the risk of CVD by reducing the non-HDL cholesterol (Fig. 1) (16).

Considering the importance of the association between vitamin D and CVD (Fig. 2), it is noteworthy that almost 1 billion individuals worldwide are likely to be deficient in or have insufficient vitamin D, in accordance with the definition of the Institute of Medicine (Washington, WA, USA), which considers circulating 25-hydroxyvitamin D levels of $>50 \mathrm{nmol} / \mathrm{l}$ indicative of vitamin D deficiency (5). Even in Western countries such as the United States, childhood vitamin D deficiency is as high as $9-18 \%$ (17). Although the metabolically active form of vitamin $\mathrm{D}$ is 1,25-dihydroxycholecalciferol, its half life is much shorter than its precursor, 25-hydroxyvitamin $\mathrm{D}$ and because of this the circulating levels of 25-hydroxyvitamin D are considered indicative of vitamin D nutritional status (18). A significant amount ( 90\%) of circulating 25-hydroxyvitamin D is bound to vitamin D binding protein in serum, which is useful in transporting this vitamin to different tissues. Several mutations in the gene coding for vitamin D binding protein have been identified that potentially alter its affinity for 25-hydroxyvitamin D and thus its ability to transport the vitamin in the body (19). The National Health and Nutrition Examination Survey study (Atlanta, GA, USA), with $>16,600$ subjects, suggested a strong association between vitamin $\mathrm{D}$ deficiency and angina, myocardial infarction and stroke (20). The insufficient intake of vitamin D and reduced blood levels of 1,25-dihydroxyvitamin D3 were shown to predict acute myocardial infarction and stroke after a 10 -year follow-up in the elderly population (21).

\section{Inflammation and vitamin D status}

Anti-inflammatory effects of vitamin D have been shown to be beneficial in the treatment of congestive heart failure in children. In a double-blind study, it was demonstrated that a 12-week vitamin D supplementation to infants with conges- 


\section{Molecular events leading to cardiac and vascular dysfunction during reduced 1. 25-dihydroxyvitamin D availability}

Reduced 25-hydroxyvitamin D in blood

Reduced 1. 25-dihydroxyvitamin D3 availability

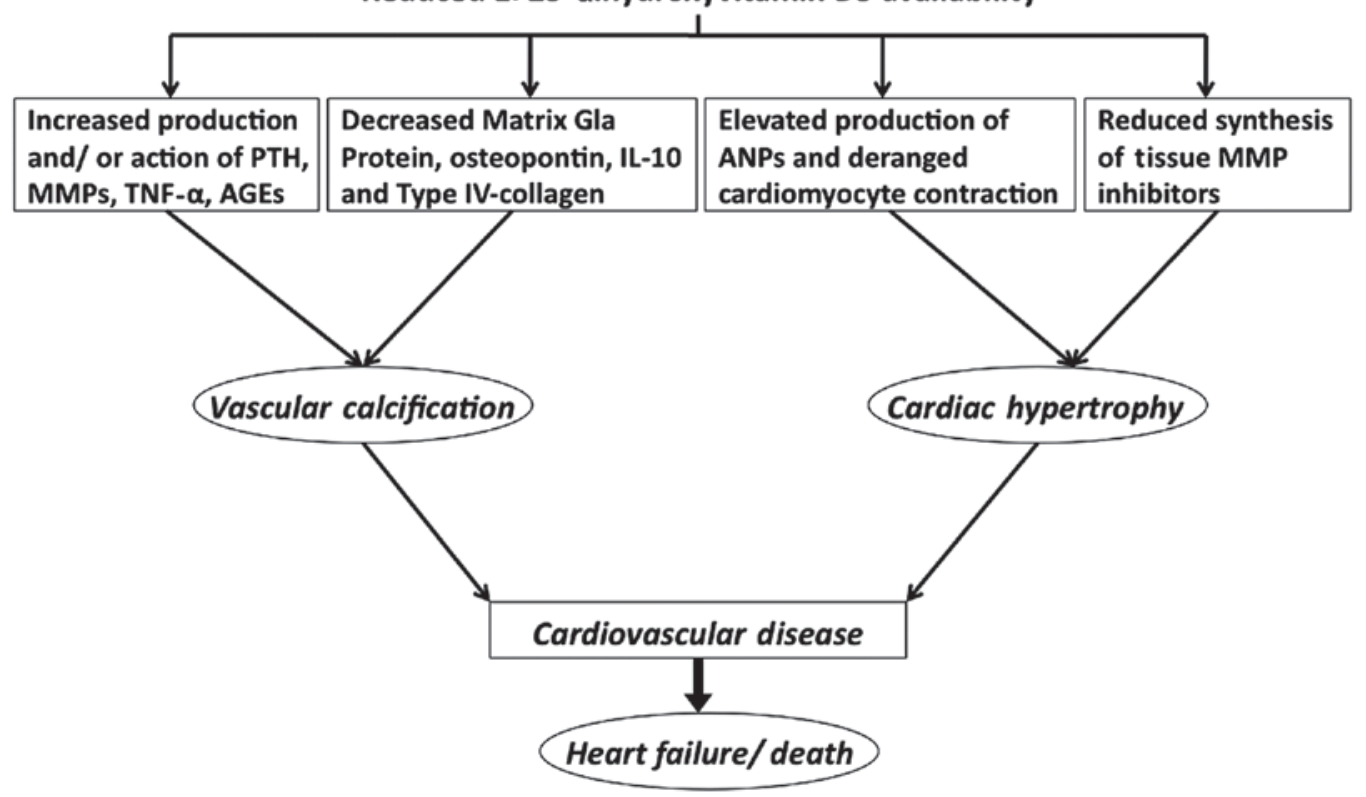

Figure 2. Molecular events leading to cardiac and vascular dysfunction during reduced 1,25-dihydroxyvitamin D availability. Scheme showing different biochemical events underlying the clinical manifestations of vitamin D deficiency and reduced availability of 1,25-hydroxyvitamin D3. PTH, parathyroid hormone; MMP, matrix metalloproteinase; TNF- $\alpha$, tumor necrosis factor- $\alpha$; AGEs, advanced glycation end-products; ANP, atrial natriuretic peptide.

tive heart failure secondary to dilated cardiomyopathy or congenital heart disease with systemic left ventricular systolic dysfunction, led to a statistically significant increase in the anti-inflammatory cytokine interleukin (IL)-10, while the proinflammatory cytokines IL- 6 and tumor necrosis factor- $\alpha$ (TNF- $\alpha$ ) were decreased as compared to the placebo control group, comprising of similar patients. These beneficiary changes in IL-10 levels in vitamin D-treated infants were accompanied with echocardiographic improvements in left ventricular dimensions and systolic function indices. The abovementioned studies are an important indication of the therapeutic benefit of vitamin D in controlling paediatric congestive heart failure (22).

Children with Kawasaki disease have been found to suffer from severely reduced 25-hydroxyvitamin D levels compared to healthy controls (23). Patients with Kawasaki disease were found to have elevated serum levels of many proinflammatory cytokines, including IL-6 and TNF- $\alpha$, which trigger vascular endothelial cell activation and vascular wall inflammation. Notably, 1,25-dihydroxyvitamin D3 inhibits TNF- $\alpha$-induced vascular cell adhesion molecule-1 expression and IL- 8 production thus regulating the inflammatory response in human coronary arterial endothelial cells (24). Several studies have also shown low circulating vitamin D levels in patients with a wide variety of inflammatory conditions, suggesting a strong association between vitamin D status and inflammation-mediated disease processes. There is a strong possibility that 25-hydroxyvitamin D deficiency plays a role in the development of CVD in patients with Kawasaki disease (25).

\section{Vitamin D and metabolic disease}

Finding of previous studies have indicated that 25-hydroxyvitamin D3 (derived from animal sources, in particular by sunlight radiation on skin) but not 25-hydroxyvitamin D2 (derived from plant steroids), is positively associated with HDL-cholesterol in children (9). This is noteworthy because these two forms are converted to the metabolically active vitamin D form in the kidneys. Additionally, there is an apparent lack of association between circulating 25-hydroxyvitamin D2 and many cardiovascular risk factors, whereas a strong inverse correlation between 25-hydroxyvitamin D3 levels and fasting insulinemia was identified (26). Thus, elevated 25-hydroxyvitamin D3 in children reduces insulin resistance, an important risk factor for diabetes and possibly for CVD. Another study revealed genetic evidence for a causal relationship between elevated blood levels of 25-hydroxyvitamin D and reduced blood pressure and risk of hypertension (27).

Few clinical trial studies addressing vitamin D supplementation on childhood cardiovascular events are available. In a randomized controlled trial in 14- to 18-year-old healthy African-American youths, vitamin D administration was shown to antagonize the progression of aortic stiffness and to reduce adiposity, an important risk factor for CVDs (28). In another larger study involving 247 obese children and adolescents aged 8-16 years, it was observed that almost $47 \%$ of the children were vitamin D-deficient and that there was a strong correlation of low levels of vitamin D with increased carotid intima media thickness and metabolic syndrome, idependent of other 
traditional risk factors of atherosclerosis (29). In a vitamin D deficiency treatment study involving 14 post-menarcheal obese females, no association was identified between vitamin D level and blood pressure. Nevertheless, an improvement of glucose homeostasis in these subjects was observed (30). In a study on 4- to 16-year-old Turkish pre-pubertal children (31), $98 \%$ of patients were vitamin $\mathrm{D}$ deficient $(<20 \mathrm{ng} / \mathrm{ml})$. However, no relationship between blood 25-hydroxyvitamin D3 levels and body-mass index was observed. However, there was an inverse correlation between HOMA-insulin resistance and serum 25-hydroxyvitamin D3. Female children were also more likely to be vitamin D-deficient.

\section{Vitamin deficiency in CKD}

CKD has been known to be associated with CVDs in adults and LVH, which develops in the early stages of CKD, and it is an independent risk factor for mortality with CKD (32). In a recent study on pediatric patients with CKD, the relationship between parameters of calcium-phosphorous metabolism and the status of 25-hydroxyvitamin D and cardiovascular structure and function were examined (2). The present findings showed that 25-hydroxyvitamin D status and systolic blood pressure are independent predictors of increased left ventricular mass, whereas diastolic dysfunction can be predicted by the PTH level. The elevated left ventricular mass occured because of the increased after-load on the left ventricle caused by systolic hypertension in these patients. Thus, in pediatric CKD patients there is an association of nutritional vitamin $\mathrm{D}$ deficiency with increased left ventricular mass and diastolic dysfunction. Considering the majority of cardiovascular changes cannot be reverted, early intervention with vitamin D supplementation in children is the best preventive measure.

In conclusion, Vitamin D nutritional deficiency or even insufficiency, particularly in children is associated with rickets and disturbed bone and calcium metabolism, as well as inflammatory diseases and progressive CVDs. Low levels of 25-hydroxyvitamin D occur due to reduced intake as well as because of geographical location, leading to diseases such as CKD. Childhood vitamin D deficiency can progress and lead to atherosclerosis and other CVDs in adulthood. Early intervention with vitamin D supplementation is an ideal approach towards preventive therapy.

\section{References}

1. Mathers CD and Loncar D: Projections of global mortality and burden of disease from 2002 to 2030. PLoS Med 3: e442, 2006.

2. Patange AR, Valentini RP, Gothe MP, Du W and Pettersen MD: Vitamin D deficiency is associated with increased left ventricular mass and diastolic dysfunction in children with chronic kidney disease. Pediatr Cardiol 34: 536-542, 2013.

3. Berenson GS, Srinivasan SR, Bao W, Newman WP III, Tracy RE and Wattigney WA: Association between multiple cardiovascular risk factors and atherosclerosis in children and young adults. The Bogalusa Heart Study. N Engl J Med 338: 1650-1656, 1998.

4. Lauer RM and Clarke WR: Use of cholesterol measurements in childhood for the prediction of adult hypercholesterolemia. The Muscatine Study. JAMA 264: 3034-3038, 1990.

5. Holick MF: Vitamin D deficiency. N Engl J Med 357: 266-281, 2007.

6. Rose G: Cold weather and ischaemic heart disease. Br J Prev Soc Med 20: 97-100, 1966.
7. Fleck A: Latitude and ischaemic heart disease. Lancet 1: 613, 1989.

8. Wang L, Song Y, Manson JE, Pilz S, März W, Michaëlsson K, Lundqvist A, Jassal SK, Barrett-Connor E, Zhang C, et al: Circulating 25-hydroxy-vitamin D and risk of cardiovascular disease: a meta-analysis of prospective studies. Circ Cardiovasc Qual Outcomes 5: 819-829, 2012.

9. Williams DM, Fraser A, Sayers A, Fraser WD, Hingorani A, Deanfield J, Davey Smith G, Sattar N and Lawlor DA: Associations of 25-hydroxyvitamin D2 and D3 with cardiovascular risk factors in childhood: Cross-sectional findings from the Avon Longitudinal Study of Parents and Children. J Clin Endocrinol Metab 97: 1563-1571, 2012.

10. Andersson P, Rydberg E and Willenheimer R: Primary hyperparathyroidism and heart disease - a review. Eur Heart J 25: 1776-1787, 2004

11. Xiang W, Kong J, Chen S, Cao LP, Qiao G, Zheng W, Liu W, Li X, Gardner DG and Li YC: Cardiac hypertrophy in vitamin D receptor knockout mice: role of the systemic and cardiac renin-angiotensin systems. Am J Physiol Endocrinol Metab 288: E125-E132, 2005.

12. Lind L, Hänni A, Lithell H, Hvarfner A, Sörensen $\mathrm{OH}$ and Ljunghall S: Vitamin D is related to blood pressure and other cardiovascular risk factors in middle-aged men. Am J Hypertens 8: 894-901, 1995.

13. O'Connell TD, Berry JE, Jarvis AK, Somerman MJ and Simpson RU: 1,25-Dihydroxyvitamin D3 regulation of cardiac myocyte proliferation and hypertrophy. Am J Physiol 272: H1751-H1758, 1997.

14. Bae S, Singh SS, Yu H, Lee JY, Cho BR and Kang PM: Vitamin D signaling pathway plays an important role in the development of heart failure after myocardial infarction. J Appl Physiol (1985) 114: 979-987, 2013.

15. Raitakari OT, Juonala M, Kähönen M, Taittonen L, Laitinen T, Mäki-Torkko N, Järvisalo MJ, Uhari M, Jokinen E, Rönnemaa $\mathrm{T}$, et al: Cardiovascular risk factors in childhood and carotid artery intima-media thickness in adulthood: the Cardiovascular Risk in Young Finns Study. JAMA 290: 2277-2283, 2003.

16. Birken CS, Lebovic G, Anderson LN, McCrindle BW, Mamdani M, Kandasamy S, Khovratovich M, Parkin PC and Maguire JL; TARGet Kids! collaboration: Association between Vitamin D and Circulating Lipids in Early Childhood. PLoS One 10: e0131938, 2015.

17. Karalius VP, Zinn D, Wu J, Cao G, Minutti C, Luke A, Kramer H and Durazo-Arvizu R: Prevalence of risk of deficiency and inadequacy of 25-hydroxyvitamin D in US children: NHANES 2003-2006. J Pediatr Endocrinol Metab 27: 461-466, 2014.

18. Jones G: Pharmacokinetics of vitamin D toxicity. Am J Clin Nutr 88: 582S-586S, 2008.

19. Carpenter TO, Zhang JH, Parra E, Ellis BK, Simpson C, Lee WM, Balko J, Fu L, Wong BY and Cole DE: Vitamin D binding protein is a key determinant of 25-hydroxyvitamin D levels in infants and toddlers. J Bone Miner Res 28: 213-221, 2013.

20. Kendrick J, Targher G, Smits G and Chonchol M: 25-Hydroxyvitamin D deficiency is independently associated with cardiovascular disease in the Third National Health and Nutrition Examination Survey. Atherosclerosis 205: 255-260, 2009.

21. Marniemi J, Alanen E, Impivaara O, Seppänen R, Hakala P, Rajala $\mathrm{T}$ and Rönnemaa T: Dietary and serum vitamins and minerals as predictors of myocardial infarction and stroke in elderly subjects. Nutr Metab Cardiovasc Dis 15: 188-197, 2005.

22. Shedeed SA: Vitamin D supplementation in infants with chronic congestive heart failure. Pediatr Cardiol 33: 713-719, 2012.

23. Stagi S, Rigante D, Lepri G, Matucci Cerinic M and Falcini F: Severe vitamin D deficiency in patients with Kawasaki disease: a potential role in the risk to develop heart vascular abnormalities? Clin Rheumatol: May 22, 2015 (Epub ahead of print).

24. Kudo K, Hasegawa S, Suzuki Y, Hirano R, Wakiguchi H, Kittaka $\mathrm{S}$ and Ichiyama T: 1 $\alpha, 25$-Dihydroxyvitamin $\mathrm{D}(3)$ inhibits vascular cellular adhesion molecule-1 expression and interleukin-8 production in human coronary arterial endothelial cells. J Steroid Biochem Mol Biol 132: 290-294, 2012.

25. Jamieson N and Singh-Grewal D: Kawasaki disease: A clinician's update. Int J Pediatr 2013: 645391, 2013. 
26. Williams DM, Fraser A, Sayers A, Fraser WD, Hyppönen E, Smith GD, Sattar N and Lawlor DA: Associations of childhood 25-hydroxyvitamin D2 and D3 and cardiovascular risk factors in adolescence: prospective findings from the Avon Longitudinal Study of Parents and Children. Eur J Prev Cardiol 21: 281-290, 2014.

27. Vimaleswaran KS, Cavadino A and Berry DJ: Association of vitamin $\mathrm{D}$ status with arterial blood pressure and hypertension risk: a mendelian randomisation study. Lancet Diabetes Endocrinol 2: 719-729, 2014.

28. Dong Y, Stallmann-Jorgensen IS, Pollock NK, Harris RA, Keeton D, Huang Y, Li K, Bassali R, Guo DH, Thomas J, et al: A 16-week randomized clinical trial of 2000 international units daily vitamin D3 supplementation in black youth: 25-hydroxyvitamin D, adiposity, and arterial stiffness. J Clin Endocrinol Metab 95: 4584-4591, 2010
29. Atabek ME, Eklioglu BS, Akyürek N and Alp H: Association between vitamin D level and cardiovascular risk in obese children and adolescents. J Pediatr Endocrinol Metab 27: 661-666, 2014

30. Ashraf AP, Alvarez JA, Gower BA, Saenz KH and McCormick KL: Associations of serum 25-hydroxyvitamin D and components of the metabolic syndrome in obese adolescent females. Obesity (Silver Spring) 19: 2214-2221, 2011.

31. Aypak C, Türedi O and Yüce A: The association of vitamin D status with cardiometabolic risk factors, obesity and puberty in children. Eur J Pediatr 173: 367-373, 2014.

32. Gaita D, Mihaescu A and Schiller A: Of heart and kidney: A complicated love story. Eur J Prev Cardiol 21: 840-846, 2014. 